\title{
Decipher Prostate Cancer Test
}

National Cancer Institute

\section{Source}

National Cancer Institute. Decipher Prostate Cancer Test. NCI Thesaurus. Code

C128181.

A proprietary genetic test that evaluates a prostate cancer tumor for its potential for metastasis. Decipher measures the expression levels of 22 RNA biomarkers involved in multiple biological pathways that are associated with metastasis and aggressive prostate cancer to classify tumors as high, average, or low risk of metastasis. 